\title{
Proton NMR spin-diffusion studies of PS-PB block copolymers at low field: two- vs three-phase model and recalibration of spin-diffusion coefficients
}

\author{
Henriette W Meyer, Horst Schneider and Kay Saalwächter
}

Low-field proton nuclear magnetic resonance (NMR) methods were used to assess the phase fractions, domain thicknesses, $T_{1}$ relaxation properties and spin diffusion (SD) coefficients $D$ of different phases in nanophase-separated polystyrenepolybutadiene block copolymers. At low field, SD experiments are challenged by rather short longitudinal relaxation times $\left(T_{1}\right)$, requiring careful consideration of the interplay of $T_{1}$ relaxation and SD effects. Building on earlier work, we used a numerical fitting procedure for a separate as well as combined analysis of phase-resolved rigid- and mobile-phase filtered SD, as well as saturation recovery curves taken on a well-defined lamellar sample. We demonstrate the advantages in using three-component model, distinguishing a rigid and a mobile, as well as an interphase that can be resolved by fits to the refocused free-induction decay. We further use domain sizes from small-angle X-ray scattering as a gauge and find that SD coefficients from literature calibrations are overestimated. Under static low-field conditions, $D$ for the rigid polystyrene phase is found to be $0.38 \pm 0.06 \mathrm{~nm}^{2} \mathrm{~ms}^{-1}$, and we propose a rescaling of a literature calibration correlating $D$ for the mobile phase with its $T_{2}$ relaxation time.

Polymer Journal (2012) 44, 748-756; doi:10.1038/pj.2012.88; published online 13 June 2012

Keywords: block copolymers; domain sizes; low-field NMR; NMR relaxation; spin diffusion; time-domain NMR

\section{INTRODUCTION}

Many macroscopical features of polymers, such as their mechanical deformation properties, are a direct result of their microscopic, molecular and nanoscale characteristics. Therefore, investigations of the structure and phase composition of heterogeneous polymer systems at the nanoscale are of high relevance. Owing to their robustness and easy availability, low-cost low-field nuclear magnetic resonance (NMR) methods are particularly versatile for the assessment of the segmental and chain dynamics, as well as potential inhomogeneities, as for instance arising in inhomogeneous polymer networks, nanophase-separated heteropolymers or semicrystalline homopolymers. ${ }^{1}$

The amount of the different components, their mobility and morphology can be studied, where in particular, the latter can be addressed by employing spin-diffusion (SD) techniques. ${ }^{2-5}$ In this way, domain sizes in heterogeneous materials are accessible on a length scale of $0.5-200 \mathrm{~nm}^{2}$ Such experiments are based upon the distinction of different phases by their mobility, first in order to quantify their relative amount and second to selectively polarize them. With a gradient in magnetization established, the SD process will lead to its reequilibration over the sample. Both the SD process and the proton NMR time-domain signal are mainly influenced by the strong ${ }^{1} \mathrm{H}^{-1} \mathrm{H}$ dipole-dipole coupling (DDC). Because of its orientation dependence, dynamics has a strong effect on the effective DDC and thus on the SD coefficient and on the shape of the free-induction decay (FID), the latter allowing for a distinction of mobile and more rigid phases. ${ }^{4,5}$

One major problem of low-field SD experiments is the rather short $T_{1}$ relaxation time in particular of mobile polymer phases, requiring in-depth investigations of the interplay of $T_{1}$ relaxation and $\mathrm{SD}$ effects, because the former competes with the latter in the same experiment, leading to intensity decay at long SD (mixing) times $t_{\text {diff }}$ The process of SD out of a region of length $d$ with a constant diffusion coefficient $D$ has a characteristic diffusion time $\tau_{\text {diff }}=d^{2} / D$. Starting with a nonequilibrium distribution of magnetization, SD dominates the evolution of magnetization for $t_{\text {diff }} \leqslant \tau_{\text {diff }}=d^{2} / D \ll T_{1},{ }^{2}$ and only for very large mixing times, the $T_{1}$-relaxation process dominates. With $T_{1}$ of the order of $10 \mathrm{~ms}$, its effect on the SD process during $t_{\text {diff }}$ in our used polymer systems, having typical domain sizes of approximately $10 \mathrm{~nm}$, has to be considered explicitly. ${ }^{5}$ A simple overall up-correction of the intensity is not possible because the different regions have largely different $T_{1}$ s. Although analytical solutions for $\mathrm{SD}$ in one to three dimensions are available, ${ }^{6-8}$ a closed-form treatment including $T_{1}$ effects is not possible.

In a previous publication, ${ }^{5}$ we have addressed this problem with a dedicated fitting program based on a simulation of the SD process by 
finite step integration of the combined $\mathrm{SD}$ and relaxation rate equation on a lattice reflecting the sample morphology. Combining $\mathrm{SD}$ and $T_{1}$ relaxation, the rate equation for the magnetization $M(r, t)$ reads:

$$
\frac{\partial M(r, t)}{\partial t}=D\left[\frac{\partial^{2} M(r, t)}{\partial^{2} r^{2}}\right]+\frac{1}{T_{1}}\left[M_{0}-M(r, t)\right]
$$

where $M_{0}$ is the equilibrium magnetization. With constant diffusion coefficients $D$ and relaxation times $T_{1}$ within individual domains, Equation (1) can be solved for each region, using cyclic boundary conditions to correctly represent for instance lamellar systems. Equating the integrated magnetization of a specific subphase with the measured phase-resolved signal $I\left(t_{m}\right) \propto \int M\left(r, t_{m}\right) d r$, an interactive fitting procedure to actual data yields the domain sizes and the $T_{1}$ times. Alternatively, for given domain sizes determined by other means, $D$ can be estimated.

Our previous work ${ }^{5}$ was restricted to fits to a two-phase model distinguishing a mobile and a rigid phase, the latter, however, comprising another two spectroscopically distinguishable components, a rigid glassy and an interphase fraction. We were able to establish some empirical rules to correct for effects of $T_{1}$ relaxation in order to be able to apply the simple initial-rate approximation, allowing for an estimation of the sizes of the mobile and combined rigid phases based upon a linear extrapolation of the (corrected) SD data. ${ }^{2,3}$

In this work, we apply the same proton NMR methods in order to identify the phase fractions $f$, the domain thicknesses $d$, the longitudinal $\left(T_{1}\right)$ and transverse $\left(T_{2}\right)$ relaxation properties and the SD coefficients $D$ of different phases in polystyrene-polybutadiene (PSPB) di- and multiblock copolymers. The new point is that we assess the feasibility of a three-phase model not only in fitting the FID data but also in the numerical fitting of the SD data, comparing the results with the analysis based on the two-phase model. We further assess the absolute values of the phase-resolved SD coefficients $D$, using results for the long period of our lamellar systems as obtained by small-angle $\mathrm{X}$-ray scattering (SAXS) measurements.

\section{EXPERIMENTAL PROCEDURE}

\section{Samples}

In this work, two PS-PB block copolymer samples were investigated; first, a well-defined linear diblock copolymer with a molecular weight of $20 \mathrm{~kg} \mathrm{~mol}^{-1}$ and polydispersity $\leqslant 1.1$ termed SB (Polymer Standards Service, Mainz, Germany), and second as a reference, a sample studied already in previous papers, ${ }^{5,9,10}$ namely a star block copolymer $\left(M=99 \mathrm{~kg} \mathrm{~mol}^{-1}\right.$, polydispersity $\approx 2.1$ ) with $\mathrm{PB}$ as four-arm star core and PS chains of different length as arm ends. This sample is referred to as KR (K-Resin KR03; Chevron Phillips Chemical Company LLC, Overijse, Belgium). Both systems have a lamellar phase-separated morphology.

As shown by transmission electron microscopy for $\mathrm{KR}$, the $\mathrm{PB}$ core forms disordered lamellae with a regular width of about $10 \pm 1 \mathrm{~nm} .{ }^{5}$ A partial mobilization and mixing of the PS block into the soft PB block at the interface was evidenced by high-resolution magic-angle spinning (MAS)-NMR measurements over a large temperature range. ${ }^{9}$ The volume fraction of $\mathrm{PB}$ units in the PB-dominated phase is around $95 \%$ at $298 \mathrm{~K}$ and $91 \%$ at $336 \mathrm{~K}$, with the mobilized PS mainly located at the interface.

Here, measurements were performed over a temperature range $300-360 \mathrm{~K}$, which is below the glass transition temperature $\left(T_{\mathrm{g}}\right)$ of PS $\left(T_{\mathrm{g}, \mathrm{PS}}=388 \mathrm{~K}\right)$ and well above the glass transition temperature of $\mathrm{PB}\left(T_{\mathrm{g}, \mathrm{PB}}=165 \mathrm{~K}\right)$. In the range between 300 and $360 \mathrm{~K}, \mathrm{~PB}$ is rubbery, whereas PS is glassy. Hence, the two phases can be distinguished at low-field NMR because of their different molecular mobility, as seen in Figure 1a. The mobile-phase contents cover ranges of $30-38 \%(\mathrm{KR})$ and $45-57 \%(\mathrm{SB})$. In the same temperature range, the samples as a whole do not melt or show any major structural rearrangements,
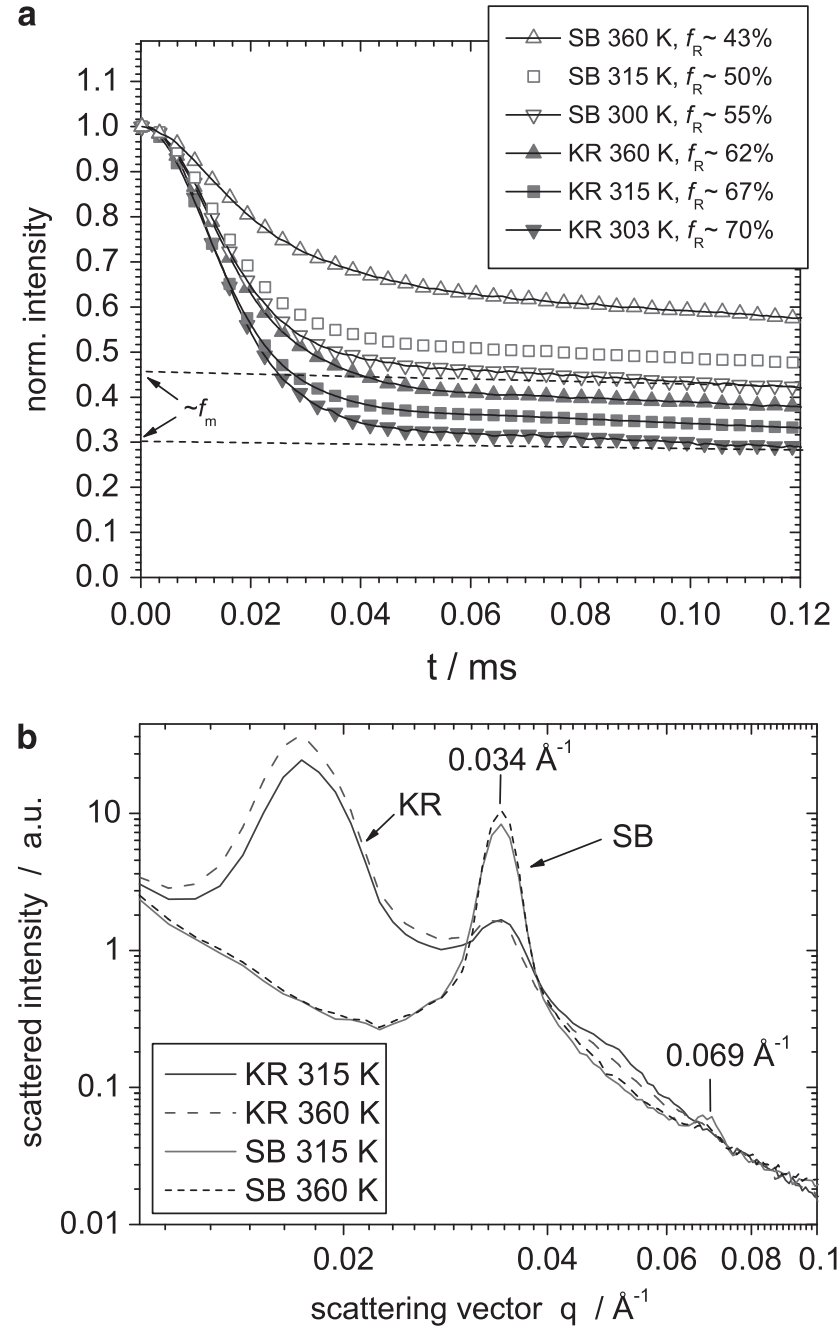

Figure 1 (a) MSE-FIDs of the SB and KR samples acquired at $300-360 \mathrm{~K}$. With increasing temperature, the fraction of the mobile phase increases, as can be seen by the increase of the slowly relaxing contribution to the FIDs. Its fraction can be estimated by simple linear back extrapolation, as indicated by the dashed lines. For SB, the mobile fraction is up to $19 \%$ larger than for KR. (b) SAXS measurements (scattering intensity vs scattering vector $q$ ) of $\mathrm{SB}$ and $\mathrm{KR}$ at 315 and $360 \mathrm{~K}$. As judged from the almost constant positions of the first maxima, the long period $(18.5 \mathrm{~nm}$ for $\mathrm{SB}$ and $36 \mathrm{~nm}$ for $\mathrm{KR}$ ) does not change upon increasing the temperature, proving that there is no major structural rearrangement. A full color version of this figure is available at Polymer Journal online.

as proven by SAXS measurements (see Figure $1 \mathrm{~b}$ ). We derived long periods of $36 \mathrm{~nm}$ for KR and $18.5 \mathrm{~nm}$ for SB.

The mass densities are $1.05 \mathrm{~g} \mathrm{~cm}^{-3}$ for PS and $0.89 \mathrm{~g} \mathrm{~cm}^{-3}$ for PB and the corresponding proton (spin) densities are 0.081 and $0.100 \mathrm{~g} \mathrm{~cm}^{-3}$ for PS and $\mathrm{PB}$, respectively. ${ }^{5}$ These parameters are needed for the evaluation of the weight and volume fractions of the individual phases from proton NMR intensities.

\section{NMR principles and experiments}

As mentioned in the Introduction, the shape of the proton NMR time-domain signal is mainly influenced by the strong and orientation-dependent ${ }^{1} \mathrm{H}-{ }^{1} \mathrm{H}$ DDC, and therefore gives information about the molecular dynamics. This is because the high mobility in a polymer phase far above its $T_{\mathrm{g}}$ is associated with continuous changes of the DDC. Because the orientation dependence of the DDC (following the second Legendre polynomial $P_{2}(\cos \theta)$ ) is such that its average over an isotropic distribution of orientations $\theta$ of the $\mathrm{H}-\mathrm{H}$ vectors with respect to the magnetic field is zero, the effective DDC is averaged to 
near-zero values if the dynamics is fast as compared with the inverse DDC constant $(\ll 20 \mu \mathrm{s})$. Note that for polymer chains, whose ends are usually fixed or at least topologically constrained by entanglements, the effective DDC is still finite. The resulting small effective DDC is associated with an accordingly longer apparent transverse relaxation time $T_{2 \mathrm{~m}}$. Note that the $T_{2 \mathrm{~m}}$ decay is often nonexponential.

Such effects of dynamics allow to distinguish the signals of the mobile and rigid phases in the FID. ${ }^{2,11}$ The strong dipolar couplings in a rigid phase induce a fast transverse relaxation of the signal with $T_{2 \mathrm{r}} \approx 20 \mu \mathrm{s}$, which is on the order of the NMR receiver dead time $\left(\tau_{\mathrm{d}} \approx 12 \mu \mathrm{s}\right.$ in our case). This part of the rigid signal has to be acquired by the aid of refocusing pulse sequences, for example, the here-used dipolar time-reversed mixed magic-sandwich echo (MSE) $)^{4,5,12,13}$ (see Figure 2c). The MSE is basically a multi-spin time-reversing pulse sequence, ${ }^{12,14}$ forming an efficient echo of almost the total signal, thus overcoming the receiver dead time and allowing for a complete acquisition of initial FID (see Figure 1a). Note that the performance of the MSE is reduced if the rigid phase features some dynamics in the intermediate $(\mathrm{kHz}-\mathrm{MHz})$ range. In such cases, a prominent one being poly(ethylene) with its intracrystalline mobility, only probe heads with a short dead time allow a quantitative analysis of the rigid phase. ${ }^{15}$

For SD experiments, see Figures $2 \mathrm{a}$ and $\mathrm{b}$, a proton-magnetization gradient has to be established, namely, one of the phases has to be selectively polarized. $\mathrm{SD}$ then proceeds during the SD (mixing) time $t_{\text {diff, followed by read-out pulse }}$ and an MSE before FID detection. A filter experiment with a dipolar 'magicand-polarization echo' (MP) filter, ${ }^{16}$ termed MP filter, is used for a selective polarization of the mobile phase. The MP filter is shown in Figure $2 a$ and explained in more detail in Mauri et al..$^{5}$ It is essentially identical to the MSE, with the exception that long filter times are realized by incrementing the interpulse spacing $\tau_{\phi}$ rather than increasing the number of pulses through $n_{\mathrm{MSE}}$, as would be more common if the MSE is used to study specific $T_{2}$ effects. The MP filter relies on the breakdown of the MSE for the case of strong DDCs.

To select the magnetization of the rigid phase, a short double-quantum (DQ) filter ${ }^{17}$ is used. The selective polarization of the rigid phase by a DQ filter is based on the excitation of DQ coherences in the rigid region on the basis of the strong DDCs. The excitation and reconversion of DQ coherences depends on the DQ excitation time $\tau_{\mathrm{DQ}}$. The selective filtering of DQ signals is based on a four-step phase cycle over one of the two pulse pairs flanking $\tau_{\mathrm{DQ}}$, as shown in Figure 2b.

All low-field NMR measurements were carried out on a Bruker (Karlsruhe, Germany) Minispec mq20 $\left(B_{0}=0.5 \mathrm{~T}\right)$ with a high-temperature probe head at $20 \mathrm{MHz}$ proton resonance frequency. The temperature was controlled by a Bruker BVT-3000 heater with airflow. The typical $90^{\circ}$ pulse length was around $2.3 \mu$ s and the relatively long dead time of the probe head was approximately $12 \mu$ s. The MP filter experiments were performed with 512 scans and a total MP filter time $\tau_{\mathrm{MP}}$ of $866 \mu \mathrm{s}$. For the measurements with the DQ filter, 512 or 1024 scans were acquired, using a $\tau_{\mathrm{DQ}}$ of $18-19 \mu$ s for which a maximal rigid-phase signal is obtained. In addition to the dedicated SD experiments, low-field saturation-recovery (SR) experiments (MSE-FID detection after a $90^{\circ}$ saturation pulse and an incremented waiting period) were performed with 2048 scans to achieve a good signal-to-noise ratio. Using many scans was generally necessary for stable fitting and thus a quantitative point-by-point evaluation of the phase fractions.

Quantitative component analysis of the NMR time-domain signals The NMR methods are based on a decomposition of the FID signal into components assigned to phases with different mobility, and rely on a quantitative detection of the quickly decaying rigid-phase signal, here realized using the MSE to overcome the receiver dead time. There is still a small loss of rigid-phase signal during the MSE due to imperfections of the pulse sequence, which has to be corrected for at all temperatures, see Mauri et al. ${ }^{5}$ for details. For KR, a nearly constant value of the relative loss of the initial signal of $\sim 14 \%$ at $300-345 \mathrm{~K}$ was noted, rising somewhat to about $17 \%$ at $360 \mathrm{~K}$, whereas for SB, a constant loss of $\sim 10 \%$, rising to $13 \%$ at $360 \mathrm{~K}$, was determined. The increase at higher temperatures is associated with the onset of motions related to the glass transition. ${ }^{1,18}$ For both samples, these relative loss of signal of the rigid phase was corrected for all MSE-detected experiments.
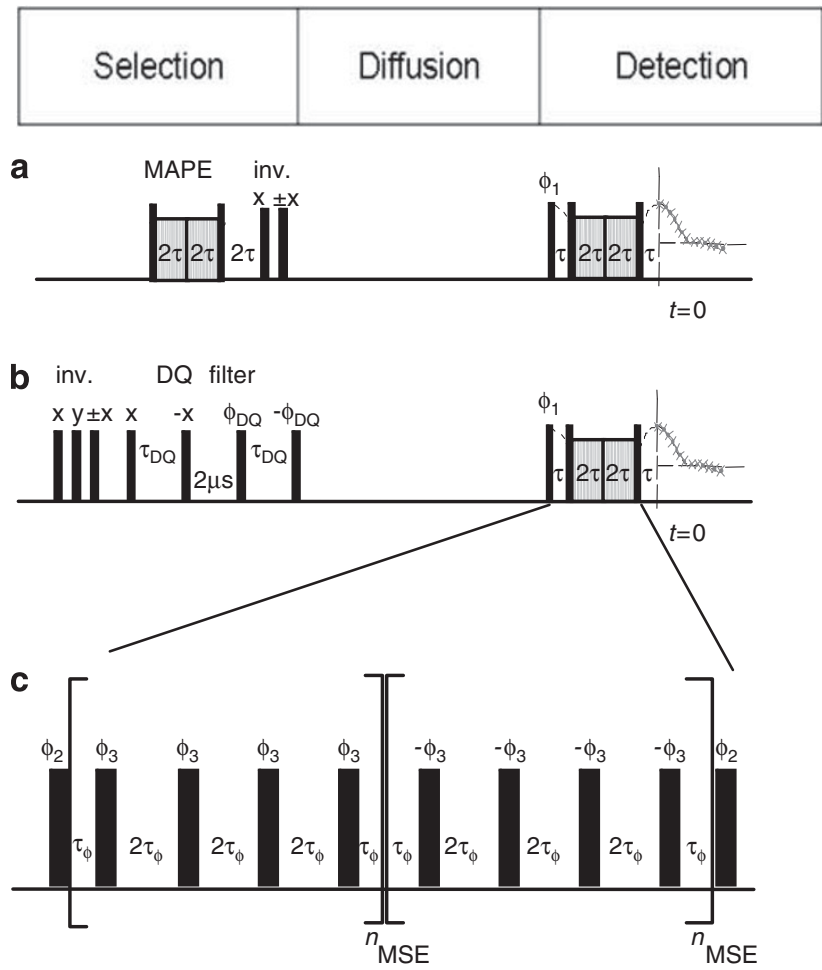

Figure 2 Spin-diffusion experiments with (a) a dipolar MP filter for selective polarization of the mobile phase. Longer filter times are realized by increasing the interpulse spacings $\tau_{\phi}$. (b) $D Q$ filter characterized by its duration $\tau_{\mathrm{DQ}}$ for selective polarization of the rigid phase. In both experiments, a MSE is applied before detection to refocus the rigid part of the signal and thus solve the dead time problem (using $n_{\mathrm{MSE}}=1$ and $a$ minimal $\tau_{\phi}$ overcome the dead time). (c) Close-up of the basic MSE/MP pulse sequence element consisting of $1090^{\circ}$ pulses. The figure is adapted from Mauri et al. ${ }^{5}$ A full color version of this figure is available at Polymer Journal online.

The components were characterized by fitting of the initial $(t \leqslant 200 \mu \mathrm{s})$ part of the MSE-FID, using a combination of modified exponential functions,

$$
\frac{F I D(t)}{F I D(0)}=f_{\mathrm{r}}\left(1-f_{\mathrm{ri}}\right) \cdot e^{-\left(t / T_{2 \mathrm{~g}}^{*}\right)^{2}}+f_{\mathrm{r}} f_{\mathrm{ri}} \cdot e^{-\left(t / T_{2 \mathrm{i}}^{*}\right)^{v_{\mathrm{i}}}}+\left(1-f_{\mathrm{r}}\right) \cdot e^{-\left(t / T_{2 \mathrm{~m}}^{*}\right)^{v_{\mathrm{m}}}}
$$

yielding the fractions of the combined rigid $\left(f_{\mathrm{r}}\right)$ and the relative intermediate phase $\left(f_{\mathrm{ri}}\right)$, as well as the apparent relaxation times $T_{2 \mathrm{~g}}{ }^{*}, T_{2 \mathrm{i}}{ }^{*}$ and $T_{2 \mathrm{~m}}{ }^{*}$ of the glassy, intermediate and mobile phases, respectively, and the exponents $v_{\mathrm{i}}$ and $v_{\mathrm{m}}$. The latter define the shape of the signal decay, which ranges from stretched exponential to Gaussian, with $0<v_{x} \leqslant 2,{ }^{4}$ with the exponent 2 for the glassy phase of course indicating the typical Gaussian signal shape of rigid organic solids. The form of Equation (2) is such that the fitting result for the combined rigid-phase fraction $\left(f_{\mathrm{r}}\right)$ is stable even when the intermediate and glassy phases are not properly determined due to low signal-to-noise. The actual fractions of the glassy, intermediate and mobile phases are obtained according to

$$
f_{\mathrm{g}}=f_{\mathrm{r}}\left(1-f_{\mathrm{ri}}\right), f_{\mathrm{i}}=f_{\mathrm{r}} \cdot f_{\mathrm{ri}}, f_{\mathrm{m}}=1-f_{\mathrm{r}}
$$

respectively. The first two equations are equivalent to

$$
f_{\mathrm{r}}=f_{\mathrm{g}}+f_{\mathrm{i}}
$$

Each initial MSE-FID was fitted only up to $0.2 \mathrm{~ms}$ to reduce the influence of the overall ill-defined shape of the FID at long times, where inhomogeneity of the magnetic field or joined effects of residual dipolar couplings, motions and inhomogeneities of the mobile-phase contribute. To ensure stable fitting, the apparent relaxation times and the shape parameters were pre-determined and fixed for the fits of mixing-time-dependant $\mathrm{SD}$ and $\mathrm{SR}$ data. $T_{2 \mathrm{~g}}{ }^{*}$ was predetermined by a Gaussian fit to a DQ-filtered FID, as shown in Figure 3. The 
parameters of the mobile phase $\left(T_{2 \mathrm{~m}}{ }^{*}\right.$ and $\left.v_{\mathrm{m}}\right)$ were obtained by fitting a MPfiltered FID, also shown in Figure 3. Along with the phase fractions for the given sample at a given temperature, the shape parameters for the interphase $\left(T_{2 \mathrm{i}}{ }^{*}\right.$ and $\left.v_{\mathrm{i}}\right)$ were determined by fitting fully relaxed MSE-FIDs, which have a good signal-to-noise ratio (1024 scans), with Equation (2). Note that the parameters and the fraction of the interphase depend on the chosen MP-filter parameter used to isolate the mobile phase. Hence, the interphase fraction and its parameters are weakly influenced by the experimental settings; they are thus operational, which cannot be avoided in a real system that exhibits a certain gradient in mobility rather than well-defined phases. We thus refrain from a discussion of the specific values, but note that usually $T_{2 \mathrm{i}}{ }^{*} \approx 25-150 \mu \mathrm{s}$ and $v_{\mathrm{i}} \approx 0.7-1.3$.

The quantitative component fractions of KR and SB, obtained by fitting of the MSE-FIDs with Equation (2) at 300-360 K, can be inspected in Table 1. Because of the mentioned field inhomogeneities, the apparent $T_{2 \mathrm{~m}}{ }^{*}$ of the mobile phases does not correspond to its real transverse relaxation time $T_{2 \mathrm{~m}}$, which has been measured by simple Hahn echo experiments (see Table 2 below). For KR, our measured $T_{2 \mathrm{~g}}{ }^{*}(\sim 18 \mu \mathrm{s}$ at $315 \mathrm{~K})$ agree well with the values determined by Mauri et al..$^{5}$ Because of the different exponents related to different magnetic field inhomogeneties, our fitted $T_{2 \mathrm{~m}}{ }^{*}$ and the ones fitted by Mauri et al. ${ }^{5}$ differ, as is expected when working with samples of different shape on different instruments. The component fractions for KR also match up well with the ones reported in earlier low-field ${ }^{5,10}$ and high-field ${ }^{9}$ NMR studies, the maximum deviation of our values to the ones in Mauri et al., ${ }^{5}$ Saalwächter et al. ${ }^{9}$ and Thomann et al. ${ }^{10}$ being about $\pm 2 \%$.

With increasing temperature, the mobile and interphase fractions rise at the expense of the rigid fraction, as shown in Table 1 and Figure 1a, where SB is seen to feature a more pronounced softening. For both samples, the decreasing rigid fraction at higher temperatures can be explained by a progressive mobilization of PS chains flanking the interphase. ${ }^{9}$ Note that the phase fractions $f_{\mathrm{g}}, f_{\mathrm{i}}$ and $f_{\mathrm{m}}$ reflect the number of protons in the phases and do not correspond to the weight fractions of the PS and PB components. To obtain proper volume fractions, the fitted fractions have to be corrected on the basis of the known respective proton densities. Thus, for the case of a onedimensional stack of flat lamellae, the ratio of the fitted phase fractions is equivalent to the ratio of the domain-thickness times and the respective spin densities.

\section{Analysis of SD and SR experiments}

To obtain the phase-resolved magnetization build-up or decay curves, for each FID in the different experiments taken as a function of $t_{\text {diff, the phase fractions }}$ were fitted with Equation (2), where the phase fractions $f_{\mathrm{r}}$ and $f_{\mathrm{ri}}$ were the only free parameters. The more meaningful fractions $f_{\mathrm{g}}$, $f_{\mathrm{i}}$ and $f_{\mathrm{m}}$ are then obtained from Equation (3). All other parameters were fixed and determined as described above. At low-field NMR, phase-resolved SR curves do not show their typical pure mono-exponential behavior, as the recovery of the magnetization is effected by relaxation and diffusion processes once one of the phases has a short $T_{1}$ on the order of $\tau_{\text {diff. }}$. The faster recovering phase then forms a source for the magnetization of the more slowly recovering phase. Fitting the SR curves with a mono-exponential function only gives apparent longitudinal relaxation times. By numerical fitting of the SR curves, possibly in combination with MP- or DQ-filtered SD curves with the simulation program, where the interplay of diffusion and relaxation is considered, the domain sizes and the real $T_{1}$ times can be estimated, see Table 3 below. For rising temperature, the longitudinal relaxation times of the rigid-phase decrease, whereas the ones of the mobile-phase increase. This behavior is the expected one, considering that the local correlation times of motion in the mobile and rigid phases are located in the slow and fast branch, respectively, of the $T_{1}$ dispersion diagram of the Bloembergen-Purcell-Pound (BPP) theory. ${ }^{11}$

The experimental DQ-filtered data in Figure 4 show that the first FIDs (at short diffusion time) contain mainly signal of the selective polarized glassy phase. Because of the lower efficiency of the DQ filter (only $<40 \%$ of the glassy-phase magnetization can be retained), the data is rather noisy, challenging a good separation of all the three phases. With increasing diffusion time, the fits give better results and $f_{\mathrm{ri}}$ as well as $f_{\mathrm{r}}$ and thus also $f_{\mathrm{g}}$ and $f_{\mathrm{m}}$, are reliably determined. In general, a precise identification of all the phase

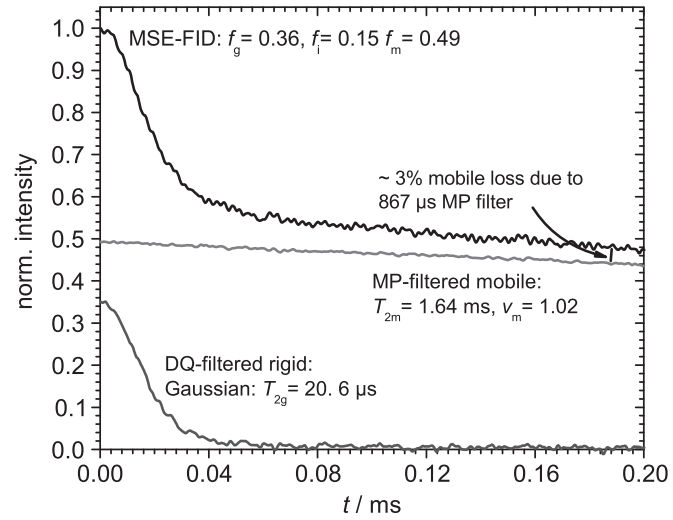

Figure 3 Decomposition of the relaxed MSE-FID signal of SB at $345 \mathrm{~K}$ by a three-component modified-exponential fit. The results of the DQ-filtered FID (scaled to match the actual $f_{\mathrm{g}}$ ) and the MP-filtered FID (on the same scale as the MSE-FID to demonstrate the negligible loss in $f_{m}$ upon filtering) are also shown, being the basis of the shape-parameter pre-determination. A full color version of this figure is available at Polymer Journal online.

Table 1 Phase fractions for KR and SB from fits to MSE-FIDs

\begin{tabular}{lrrcccc}
\hline \multicolumn{7}{c}{ KR } \\
\hline$T(K)$ & 315 & 360 & 300 & 315 & 345 & 360 \\
$f_{\mathrm{r}}(\%)$ & 67 & 62 & 55 & 49.7 & 50.3 & 43 \\
$f_{\mathrm{m}}(\%)$ & 33 & 38 & 45 & 50.3 & 49.5 & 57 \\
$f_{\mathrm{g}}(\%)$ & 60 & 52 & 51 & 46.7 & 35.5 & 23.6 \\
$f_{\mathrm{i}}(\%)$ & 6.8 & 10 & 4 & 3 & 14.8 & 19 \\
\hline
\end{tabular}

Table 2 Spin-diffusion coefficients $D$ and transverse relaxation times $T_{2}$ for the mobile phase

\begin{tabular}{lllll}
\hline$T(\mathrm{~K})$ & 300 & 315 & 345 & 360 \\
\hline $\mathrm{KR}$ & & & & \\
$T_{2 \mathrm{~m}}(\mathrm{~ms})$ & 0.68 & 0.77 & 0.95 & 1.1 \\
$D_{\mathrm{m}}\left(\mathrm{nm}^{2} \mathrm{~ms}^{-1}\right)$ & 0.33 & 0.32 & 0.29 & 0.24 \\
$\mathrm{SB}$ & & & & \\
$T_{2 \mathrm{~m}}(\mathrm{~ms})$ & 1.16 & 1.61 & 2.6 & 2.8 \\
$D_{\mathrm{m}}\left(\mathrm{nm}^{2} \mathrm{~ms}^{-1}\right)$ & 0.21 & 0.13 & 0.07 & 0.06 \\
\hline
\end{tabular}

Table 3 Results for $T_{1}$ of the different phases from DMS fits for KR and SB at 300-360 K

\begin{tabular}{lrrrrrr}
\hline & \multicolumn{3}{c}{ KR } & \multicolumn{4}{c}{$\mathrm{SB}$} \\
\hline$T(\mathrm{~K})$ & 315 & 360 & 300 & 315 & 345 & 360 \\
$T_{1 \mathrm{~g}}(\mathrm{~ms})$ & 616 & 580 & 335 & 413 & 317 & 170 \\
$T_{1 \mathrm{i}}(\mathrm{ms})$ & 144 & 192 & 300 & 265 & 225 & 232 \\
$T_{1 \mathrm{~m}}(\mathrm{~ms})$ & 77 & 134 & 60 & 75 & 146 & 180 \\
\hline
\end{tabular}

fractions in the acquired FIDs of SR or SD experiments for short $t_{\text {diff }}$ is not always possible. In MP-filtered SD experiments, the first rising parts of the interphase and the rigid phase are too small to be separated and defined precisely. Therefore, the $f_{\text {ri }}$ parameter has been set to zero for fits up to $t_{\text {diff }} \approx 15$ ms. Equation (2) ensures that this does not affect the fitted $f_{\mathrm{r}}$ fraction. 


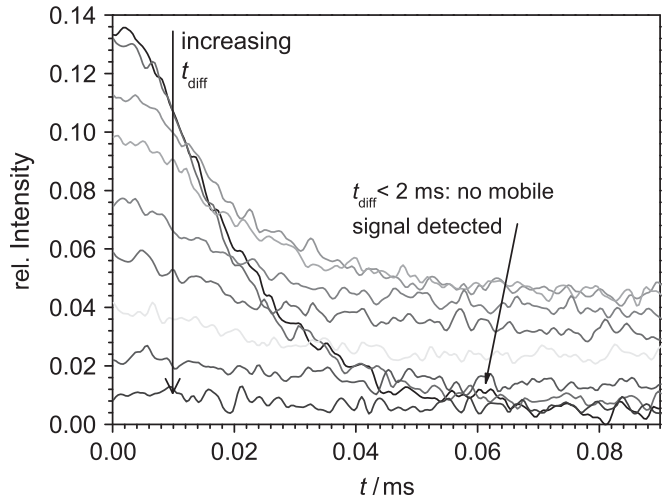

Figure 4 Selected FIDs of a DQ-filtered SD experiment with increasing SD time $t_{\text {diff }}$ for SB at $360 \mathrm{~K}$. The first FIDs (at short diffusion time) contain mainly signal of the selectively polarized glassy phase. A full color version of this figure is available at Polymer Journal online.

Note that all the intensities are referenced to the FID intensity at $t_{\text {diff }}=0$, meaning that the decay of the initial intensity by $T_{1}$ relaxation is not normalized away. In other words, the fractions do not add up to 1 at longer $t_{\text {diff }}$

\section{SD coefficients}

For the SD coefficient $D$ of the rigid phase of glassy polymers, a value of $0.8 \mathrm{~nm}^{2} \mathrm{~ms}^{-1}$ appears largely accepted. This value has been determined by SD experiments on glassy polymer samples (PS/PMMA), where the domain sizes of the rigid PS domain were known from SAXS and electron microscopical measurements. ${ }^{19}$ The SD coefficients of the mobile phase of block copolymers can be derived from a calibration published by Mellinger et al. ${ }^{3}$ based on its transverse relaxation time:

$$
D_{\mathrm{m}}\left(T_{2 \mathrm{~m}}^{-1}\right)=A \times\left(4.45 \times 10^{-5} \cdot T_{2 \mathrm{~m}}^{-1}+0.26\right) \mathrm{nm}^{2} \mathrm{~ms}^{-1} \text { for } T_{2 \mathrm{~m}}<0.9 \mathrm{~ms}
$$

$$
D_{\mathrm{m}}\left(T_{2 \mathrm{~m}}^{-1}\right)=A \times\left(8.2 \times 10^{-6} \cdot\left(T_{2 \mathrm{~m}}^{-1}\right)^{1.5}+0.007\right) \mathrm{nm}^{2} \mathrm{~ms}^{-1} \text { for } T_{2 \mathrm{~m}}>0.9 \mathrm{~ms}
$$

Note that $T_{2 \mathrm{~m}}^{-1}$ has to be given in $\mathrm{Hz}$. The prefactor $A$ is unity in the original publication, and below, its possible adjustment will be discussed.

The transverse relaxation times of the mobile phase $T_{2 \mathrm{~m}}$ were measured by Hahn echo experiments, and the resulting SD coefficients from Equations (5) and (6) are presented in Table 2. Our calculated values for KR correspond to those given by Mauri et al. ${ }^{5}$ The transverse relaxation times $T_{2 \mathrm{~m}}$ and SD coefficients $D_{\mathrm{m}}$ of KR and SB differ, as is shown by the values in Table 2. A faster and more isotropic chain dynamics reduces the residual dipolar couplings, leading to a slower transverse relaxation and a smaller $D_{\mathrm{m}}$, and thus a slower SD process within the mobile phase. We have determined the average residual dipolar coupling of the mobile phase by MP-filtered multiplequantum experiments $\mathrm{s}^{10,20}$ and found that the soft phase of SB is indeed characterized by lower residual dipolar couplings than KR. This is consistent with the $T_{2 \mathrm{~m}}$ results and confirms that chains grafted on both ends, as is the case in the multiblock structure of KR, have a more network-like and anisotropic behavior than the unilaterally grafted chains in diblock copolymers such as $\mathrm{SB}$

\section{Numerical fitting program}

The simulation program described in Mauri et al..$^{5}$ was used for numerical fitting of experimental SR and SD curves and simulations of such curves for a wide variety of parameters. The simulation program is based on a numerical solution of the diffusion equation, Equation (1), on a lattice representing lamellar systems with two or three phases. For the data-fitting process, an iterative fitting routine based on the Marquardt-Levenberg method was used. The program generally allows to simulate the diffusion process in two or three

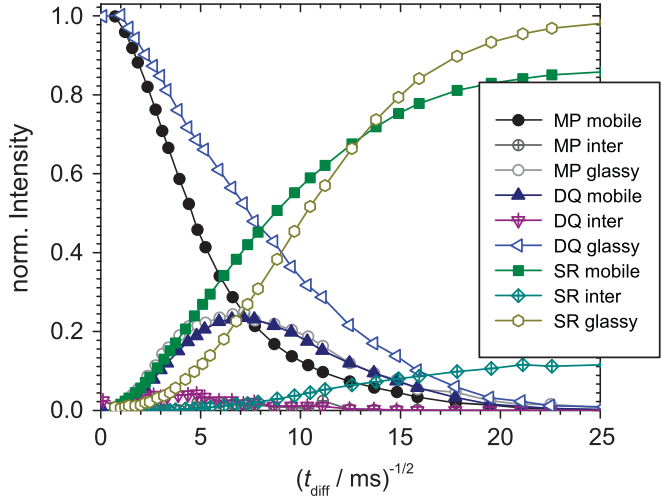

Figure 5 Comparison of DQ-filtered and MP-filtered SD curves and SR curves for $\mathrm{SB}$ at $300 \mathrm{~K}$ plotted vs $t_{\text {diff }}{ }^{1 / 2}$. They are influenced by the relaxation and SD processes in each phase. The initial part of the source curve shows a Gaussian shape, whereas the sink and SR curves have an initial sigmoidal form. The fraction of the interphase is small at $300 \mathrm{~K}$.

dimensions, and in its latest version, it considers explicitly the interphase characterized by an averaged composition (spin density) and SD coefficient. ${ }^{5,9}$

\section{RESULTS AND DISCUSSION}

\section{Comparison of the two- and three-phase model}

It is well established that block copolymers have a nonnegligible interfacial region. 5,9,10,21 From Figure 5, where SD and SR curves are plotted vs the square root of the diffusion time, a sigmoidal initial rise of the source curve is apparent. This effect is stronger for the MPfiltered SD curves than for the DQ-filtered SD curves and it is found to decrease with higher temperatures for both curves. The comparison with simulated curves has shown that the initial nonlinear rise of the curves results from a subtle yet specific interphase effect, as already discussed in Mauri et al. ${ }^{5}$ In essence, it is due to a nonpolarized region within the mobile phase close to the (detectable) interphase characterized by $f_{\mathrm{i}}$, the reason being that actual materials feature a mobility gradient rather than dynamically distinct phases. The phenomenon was taken into account in our fits and simulations by setting about $10 \%$ of the polarization of the mobile phase to zero in a small region close to the interface.

In the two-phase model used previously, ${ }^{5}$ the detectable interphase fraction $f_{\mathrm{i}}$ was considered as part of the rigid phase. The analyses presented herein are now based upon fits on the basis of a three-phase model consisting of a glassy and a mobile phase and an explicit interphase. The spin density and SD coefficients of the latter were set to the arithmetic mean values of the corresponding values of the glassy and mobile phases. The apparent $T_{2}^{*}$ times of the interphase taken from the component fits are just a bit larger than the ones of the glassy phase, indicating that SD is rather effective in this phase. However, because of its intermediate timescale mobility, it is rather efficiently suppressed by either magnetization filter.

Determination of the lamellar sizes by numerical fitting of SD data By numerical fitting of DQ- and MP-SD curves, as well as SR curves (see Figure 5), with our simulation program, the phase-resolved relaxation times, domain thicknesses and diffusion coefficients were determined. Because of scale invariance of the diffusion process, the time-dependent SD curves are identical if one changes the domain thicknesses by a factor $c$ and simultaneously the diffusion coefficients by $c^{2}$, as can be inferred from Equation (1). Thus, at least one of these 

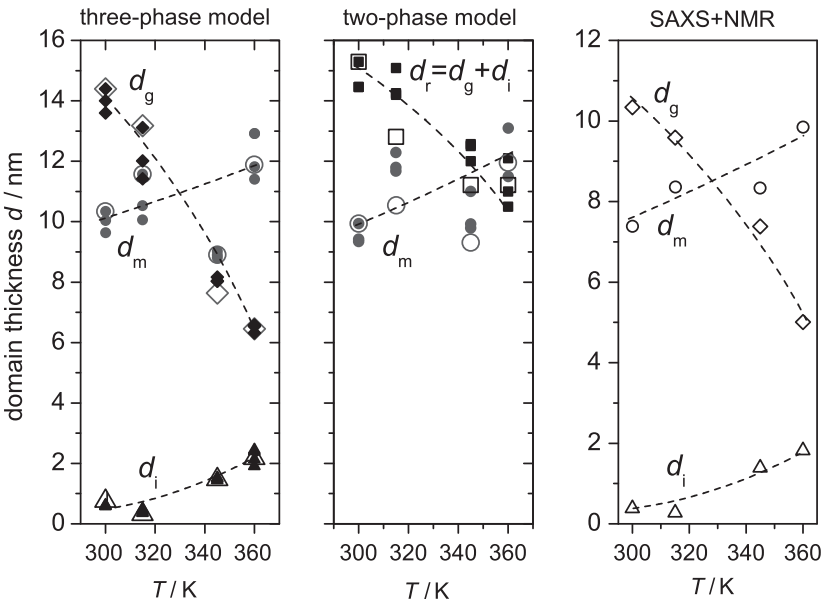

Figure 6 Temperature-dependent domain sizes for the SB sample obtained from DMS fits (large symbols) and from fits to less than three datasets (small symbols) for the three- and two-phase models (left and middle panels, respectively). The two-phase model gives only the size of the combined rigid phase $d_{\mathrm{r}}=d_{\mathrm{g}}+d_{\mathrm{i}}$. The right panel shows domain sizes calculated from the SAXS-determined long period according to Equation 7. $d_{\mathrm{g}}$, diamonds; $d_{\mathrm{m}}$, circles; $d_{\mathrm{i}}$, triangles; and $d_{\mathrm{r}}$ squares. A full color version of this figure is available at Polymer Journal online.

parameters has to be determined separately by another method and has to be fixed upon fitting.

We start with fits for which the SD coefficients were fixed at the values discussed above. The resulting $T_{1}$ relaxation times and domain sizes are shown in Table 3 and Figure 6, respectively. The former lists only results from simultaneous fits to DQ- and MP-filtered SD, as well as SR data (termed 'DMS fit': $3 \times 3$ simultaneously fitted curves in total), while the latter also shows domain sizes extracted from fits of single SR datasets, or simultaneous fits of SR curves and a single set of SD (MP or DQ) curves (MP-SD/SR and DQ-SD/SR fits, respectively). Fits to just one or two out of three datasets are intrinsically more sensitive to parameter interdependencies, in particular when the underlying (two- or three-phase) model is not accurately reflecting the actual sample. This is the reason for the scatter of the different results.

Generally, it should be stated that the most reliable results are obtained from fits that include the phase-resolved SR datasets. This is because the ratio of their limiting values at long times corresponds to the ratio of the equilibrium component proton fractions. Under the assumption of perfect lamellar morphology, the ratio of the domain volumes is directly obtained from the proton ratio by division through the corresponding spin-density ratio. Thus, relative size information is directly encoded in such data, stabilizing the fits. We have therefore not included fits to just MP- or DQ-SD curves, as they are generally less stable and reliable because of their decay to zero at long $t_{\text {diff }}$.

Taking the results of the DMS fits as a reference, we first address the temperature-dependent results for the KR sample (see Table 4). We have previously studied the domain sizes of the combined rigid and mobile phases of KR by high-field NMR, obtaining $d_{\mathrm{m}}=17 \mathrm{~nm}$ and $d_{\mathrm{r}}=42 \mathrm{~nm}$ at $309 \mathrm{~K}^{9}{ }^{9}$ Under these conditions, $T_{1}$ relaxation has virtually no influence and our present approach is nicely confirmed by the results from the DMS fits on the basis of the two-phase model at $315 \mathrm{~K}$, where $d_{\mathrm{m}}=17.2 \mathrm{~nm}$ and $d_{\mathrm{r}}=43 \mathrm{~nm}$ at $315 \mathrm{~K}$. For the thickness of the rigid domains, our values at $360 \mathrm{~K}$ are about $9 \%$
$(4 \mathrm{~nm})$ larger as compared with the ones obtained in Saalwächter et al. ${ }^{9}$ In the work of Mauri et al., ${ }^{5}$ the same low-field DQ- and MPfilter sequences, the same MSE-FID analysis method and basically the same simulation program (without full three-phase and three-dataset fitting option) were used. The $T_{1}$ relaxation times and lamellar sizes of KR obtained by the new DMS fits in the two-phase model are consistent with the values determined by DQ-SD/SR or MP-SD/SR fits in our previous work.

In general, it can be said that the obtained values from DQ-SD/SR, MP-SD/SR, SR-only and DMS fits for SB scatter appreciably less than for KR. We attribute this to the better defined structure of the diblock copolymer sample SB, as compared with the more disordered structure of KR, as evidenced by electron microscopy in Mauri et al., ${ }^{5}$ and further discussed below. We thus focus on the domain size results for SB in Figure 6, where results from all fit permutations are compiled for the two- and three-phase models. The results from latter (left panel) are seen to be on average more consistent. We take this as an indication that a three-phase model provides a more realistic account of the real spatially inhomogeneous dynamics in block copolymers. Therefore, in particular the DMS-fitting method is a reliable procedure to determine domain sizes in heterogeneous polymer systems, subject of course to a well-defined and known geometry of the diffusion process.

\section{Reevaluation of the SD coefficients}

For both samples, the long periods $(L)$ were measured by SAXS, to be compared with our NMR-determined sizes as listed in Table 4. As is seen in Figure 1b, both samples exhibit an almost negligible temperature dependence. The positions of the first- and high-order reflexes in terms of $q$, following the ratio of (1:2:3), confirm an overall lamellar arrangement in both samples. However, as judging from the different width of the reflexes and the electron microscopy evidence mentioned above, ${ }^{5}$ it is clear that SB exhibits a much better longrange lamellar order. This is of course expected considering its narrow polydispersity, as opposed to KR. ${ }^{10}$ This means that the assumption of lamellar stacks and with it the model of a purely one-dimensional $\mathrm{SD}$ process is better justified for SB.

On the basis of the one-dimensional lamellar stack model, the phase fractions determined by fitting of the equilibrated MSE-FIDs can be combined with the SAXS-determined long period to obtain the domain sizes for each phase. For example, the thickness of the glassy phase is calculated as:

$$
d_{\mathrm{g}}^{(\mathrm{S})}=\frac{\left(f_{\mathrm{g}} / \rho_{\mathrm{PS}}\right)}{\left(f_{\mathrm{g}} / \rho_{\mathrm{PS}}\right)+\left(f_{\mathrm{i}} / \rho_{\mathrm{i}}\right)+\left(f_{\mathrm{m}} / \rho_{\mathrm{PB}}\right)} \cdot L
$$

where $\rho_{x}$ is the corresponding proton densities of the phases and $L$ is the SAXS-determined long period. Because $f_{\mathrm{i}}$ is located on both sides of each lamella, $L$ corresponds to $d_{\mathrm{g}}+2 d_{\mathrm{i}}+d_{\mathrm{m}}$.

From the comparison of the sizes of the three resolved domains shown in Table 4 and Figure 6 by NMR only (left panel) and the combination of SAXS and NMR (right panel), it is apparent that the values differ by an almost constant factor. In most cases, the individual domain sizes for the different samples at the different temperatures determined by SAXS-NMR, which we consider most objective, are only $70-80 \%$ of the values obtained from NMR. Similar discrepancies were noted in Saalwächter et al. ${ }^{9}$ and Thomann et al. ${ }^{10}$ This could in principle be due to deviations from a pure onedimensional periodic lamellar structure, which could bias both methods in different ways. This is certainly in issue for KR, which was the sample previously investigated, but not for the well-ordered SB sample. 
Table 4 Lamellar sizes $d$ and SD coefficients $D$ from NMR and SAXS, based upon DMS fits

\begin{tabular}{|c|c|c|c|c|c|c|c|c|c|c|c|c|}
\hline \multirow[b]{2}{*}{$T(\mathrm{~K})$} & \multicolumn{7}{|c|}{ Three-phase model } & \multicolumn{5}{|c|}{ Two-phase model } \\
\hline & $\begin{array}{l}\mathrm{L}_{\text {tot }} \\
(\mathrm{nm})\end{array}$ & $\begin{array}{c}\mathrm{d}_{g} \\
(\mathrm{~nm})\end{array}$ & $\begin{array}{c}\mathrm{d}_{m} \\
(\mathrm{~nm})\end{array}$ & $\begin{array}{c}\mathrm{d}_{i} \\
(\mathrm{~nm})\end{array}$ & $\begin{array}{c}\mathrm{D}_{g} \\
\left(\mathrm{~nm}^{2} \mathrm{~ms}^{-1}\right)\end{array}$ & $\begin{array}{c}\mathrm{D}_{m} \\
\left(\mathrm{~nm}^{2} \mathrm{~ms}^{-1}\right)\end{array}$ & $\begin{array}{c}\mathrm{D}_{i} \\
\left(\mathrm{~nm}^{2} \mathrm{~ms}^{-1}\right)\end{array}$ & $\begin{array}{l}\mathrm{L}_{\text {tot }} \\
(\mathrm{nm})\end{array}$ & $\begin{array}{c}\mathrm{d}_{r} \\
(\mathrm{~nm})\end{array}$ & $\begin{array}{c}\mathrm{d}_{m} \\
(\mathrm{~nm})\end{array}$ & $\begin{array}{c}\mathrm{D}_{r} \\
\left(\mathrm{~nm}^{2} \mathrm{~ms}^{-1}\right)\end{array}$ & $\begin{array}{c}\mathrm{D}_{m} \\
\left(\mathrm{~nm}^{2} \mathrm{~ms}^{-1}\right)\end{array}$ \\
\hline \multicolumn{13}{|l|}{$S B$} \\
\hline $300 \mathrm{~s}$ & (18.5) & (10.34) & (7.39) & $(0.38)$ & 0.313 & 0.156 & 0.234 & (18.5) & (11.14) & $(7.36)$ & 0.437 & 0.12 \\
\hline $300 \mathrm{~N}$ & 26.2 & 14.4 & 10.3 & 0.75 & $(0.80)$ & $(0.214)$ & $(0.507)$ & 25.2 & 15.3 & 9.9 & $(0.80)$ & $(0.214)$ \\
\hline $315 \mathrm{~S}$ & $(18.5)$ & (9.58) & $(8.36)$ & $(0.28)$ & 0.318 & 0.096 & 0.207 & (18.5) & $(10.27)$ & (8.33) & 0.465 & 0.077 \\
\hline $315 \mathrm{~N}$ & 25.4 & 13.2 & 11.6 & 0.3 & $(0.80)$ & $(0.134)$ & $(0.467)$ & 23.4 & 12.8 & 10.5 & $(0.80)$ & $(0.134)$ \\
\hline $345 \mathrm{~s}$ & (18.5) & (7.38) & $(8.34)$ & $(1.4)$ & 0.444 & 0.076 & 0.26 & (18.5) & (10.3) & (8.2) & 0.665 & 0.062 \\
\hline $345 \mathrm{~N}$ & 19.5 & 7.6 & 8.9 & 1.47 & $(0.80)$ & $(0.71)$ & $(0.435)$ & 20.5 & 11.2 & 9.3 & $(0.80)$ & $(0.071)$ \\
\hline $360 \mathrm{~s}$ & $(18.8)$ & $(5.31)$ & (9.85) & $(1.82)$ & 0.452 & 0.055 & 0.253 & (18.5) & (8.95) & $(9.62)$ & 0.494 & 0.041 \\
\hline $360 \mathrm{~N}$ & 22.7 & 6.5 & 11.9 & 2.2 & $(0.80)$ & $(0.064)$ & $(0.432)$ & 23.2 & 11.2 & 12.0 & $(0.80)$ & $(0.064)$ \\
\hline \multicolumn{13}{|l|}{$K R$} \\
\hline $315 \mathrm{~S}$ & (36) & $(23.26)$ & $(10.36)$ & $(1.18)$ & 0.372 & 0.145 & 0.254 & (36) & $(25.73)$ & (10.26) & 0.286 & 0.112 \\
\hline $315 \mathrm{~N}$ & 52.7 & 33.9 & 15.3 & 1.8 & $(0.80)$ & $(0.326)$ & $(0.563)$ & 60.2 & 43.0 & 17.2 & $(0.80)$ & $(0.326)$ \\
\hline $360 \mathrm{~s}$ & (36) & $(20.3)$ & (12.17) & $(1.78)$ & 0.385 & 0.147 & 0.266 & (36) & $(24.06)$ & (11.94) & 0.302 & 0.087 \\
\hline $360 \mathrm{~N}$ & 49.4 & 27.5 & 16.6 & 2.6 & $(0.80)$ & $(0.244)$ & $(0.522)$ & 63.3 & 42.4 & 20.9 & $(0.80)$ & $(0.244)$ \\
\hline
\end{tabular}

Abbreviations: N, NMR-only results; S, SAXS-NMR combination.

For the NMR-only results (' $N$ '), the original $D$ from the $T_{2}$-based calibration were used to obtain domain sizes $d$, whereas for the SAXS-NMR combination ('S'), the domain sizes from Equation (7) were fixed and the $D$ were obtained from the fits. Values in brackets (...) were pre-determined and fixed in the respective fits.

Because of the scale invariance of the diffusion process, it can thus be assumed that the used SD coefficients are simply too large. We remind that for the glassy PS phase, an SD coefficient of $0.8 \mathrm{~nm}^{2} \mathrm{~ms}^{-1}$ was used, up to now a largely accepted value for many glassy polymers. ${ }^{3,19}$ This value was derived from SD experiments on glassy polymers (PS/PMMA), where the domain sizes were measured by SAXS and electron microscopy. ${ }^{19}$ This value was in turn also used as a basis for the calibration of the SD coefficients of the mobile phase based on $T_{2}$ times according to Mellinger et al. ${ }^{3}$ There are, however, a number of works in which significantly smaller diffusion coefficients are discussed. ${ }^{6,16,21-25}$ For example, in a paper of Schmidt-Rohr and coworkers, ${ }^{22}$ local SD processes were investigated on a scale of approximately $1 \mathrm{~nm}$, and a local SD coefficient in glassy PS of approximately $0.2 \mathrm{~nm}^{2} \mathrm{~ms}^{-1}$ was derived. This observation was recently confirmed for other systems by Roos et al. ${ }^{25}$ Vanderhart and $\mathrm{McFadden}^{23}$ suggested SD coefficients for PS on the order of $0.6 \mathrm{~nm}^{2} \mathrm{~ms}^{-1}$. Demco et al. ${ }^{16}$ calculated an SD coefficient of $0.28 \mathrm{~nm}^{2} \mathrm{~ms}^{-1}$ for crystalline poly(ethylene oxide) on the basis of the dipolar second moment of the proton lineshape.

Therefore, it seems appropriate to question the reported literature values and use our fitting program (in the three-phase version) with fixed domain sizes, taken from Equation (7), to obtain an independent estimate of the different $D_{s}$. The quality of the fits can be inspected in Figure 7. The newly determined SD coefficients are listed in Table 4 (in the lines marked by ' $\mathrm{S}$ ') and, for the case of SB, plotted in Figure 8a. For the glassy PS, we obtain an averaged $D_{\mathrm{g}} \approx 0.38 \pm 0.06 \mathrm{~nm}^{2} \mathrm{~ms}^{-1}$. Based on the scale-invariance argument, one could also take the original SD coefficients and the fit results for the domain sizes, multiplying all $D_{x}$ and $d_{x}^{2}$ by the same factor to reach the SAXS-NMR results for the latter (that is, holding the ratio $D_{x} / d_{x}^{2}$ constant for each phase), to get an independent estimate of the corrected $D_{x}$. This procedure, however, yields different results, in particular, too low corrected $D_{\mathrm{m}}$. The reason for the discrepancy is that the numerical fits yield different correction factors for $D_{\mathrm{g}}$ and $D_{\mathrm{m}}$. Although the new best-fit $D_{\mathrm{g}}$ is about $50 \%$ lower, the new $D_{\mathrm{m}}$ is only about $20-30 \%$ lower.

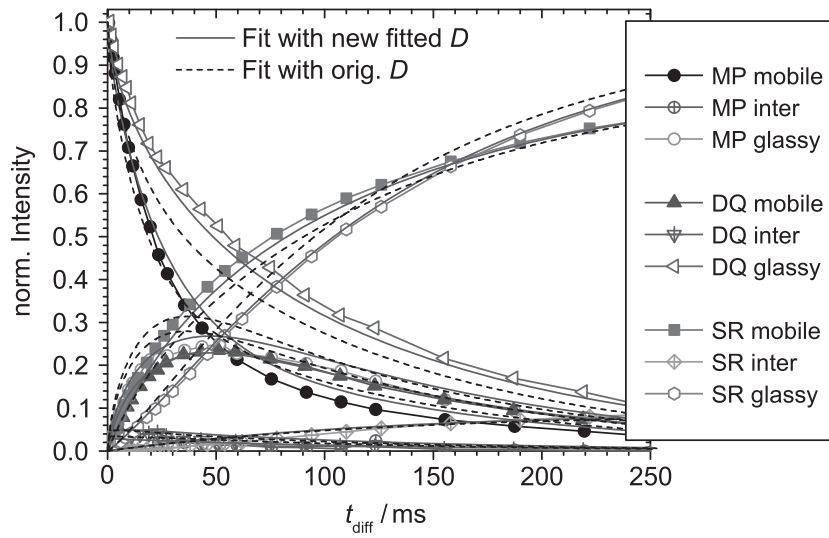

Figure 7 Numerical prediction of MP-DS, DQ-SD and SR curves with domain sizes determined by SAXS-NMR, using the original SD coefficients (dashed lines) or new SD coefficients determined by simultaneous fitting (solid lines) for SB at $300 \mathrm{~K}$. A full color version of this figure is available at Polymer Journal online.

The new diffusion coefficients of the mobile phase are smaller than the ones calculated by Mellinger et al. ${ }^{3}$ but show a similar temperature dependence. In Figure $8 \mathrm{~b}$, we plot the original $T_{2}$-based calibration, Equations (5) and (6) with $A=1$ and a corrected best-fit calibration, for which a factor of $A \approx 0.76 \pm 0.05$ was determined. As is apparent from the same Figure, the values for the KR sample deviate more strongly. As discussed above, the KR sample is more disordered, meaning that the one-dimensional lamellar stack model is not a good representation of the actual diffusion geometry in this sample. A high-dimensional diffusion geometry could explain the observed systematic trend towards lower (apparent) $D_{\mathrm{m}}$. The results for KR were thus excluded from the recalibration.

One could of course wonder why the so-determined SD coefficients differ quite substantially from the original literature values. We stress that the present work is based upon static low-field NMR, whereas in many of the cited references high-field NMR was used, possibly under 

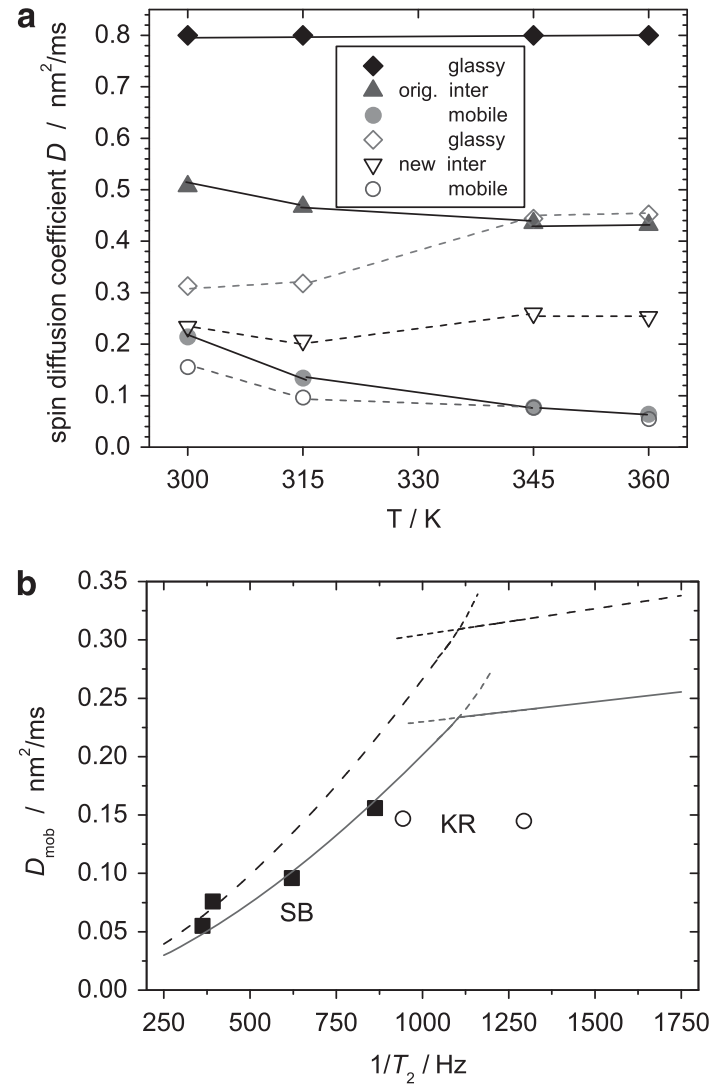

Figure 8 (a) Original SD coefficients, with $D_{m}$ taken from the literature calibration, Equations (5) and (6), as compared with new best-fit values for $\mathrm{SB}$ at 300-360 K. (b) Recalibration of Equations (5) and (6) on the basis of the $\mathrm{SB}$ results, yielding a correction factor $A \approx 0.76 \pm 0.05$ (solid line). The dashed line is the original calibration with $A=1$. A full color version of this figure is available at Polymer Journal online.

high-resolution MAS conditions. The effect of MAS is particularly interesting: Hansen $e t$ al. ${ }^{15}$ recently published a careful study of SD coefficients as a function of MAS frequency and found the expected decreasing trend with increasing MAS frequency. However, they did not compare their results with static conditions. In a recent study to be published shortly, ${ }^{26}$ it was found that locally determined SD coefficients on the basis of ${ }^{13} \mathrm{C}$-mediated hole burning ${ }^{22}$ in fact exhibit a maximum at moderate MAS on the order of a few $\mathrm{kHz}$. The original large $D_{\mathrm{g}, \mathrm{PS}} \approx 0.8 \mathrm{~nm}^{2} \mathrm{~ms}^{-1}$ was in fact determined under such conditions. ${ }^{19}$ These findings clearly call for a more in-depth study of the MAS frequency and also $B_{0}$ field dependence of SD coefficients.

\section{CONCLUSIONS}

In this work, we have shown that phase-resolved low-field ${ }^{1} \mathrm{H}$ timedomain NMR provides rich insight into the SD process within rigid and mobile domains of phase-separated polymer systems. A combination of SD and SR experiments was used to identify the phase fractions, the sizes, the $T_{1}$ relaxation times and the $\mathrm{SD}$ coefficients of the different domains, here studied for PS-PB block copolymers.

The challenging interplay of $T_{1}$ relaxation and SD effects at lowfield, arising from the rather short $T_{1}$ relaxation times of mobile phases, could be assessed with a simulation program based on the numerical solution of the diffusion equation with explicit consideration of $T_{1}$ relaxation. The $T_{1}$ values, domain sizes and/or $\mathrm{SD}$ coefficients were obtained by numerical fitting of the phase-resolved SD and SR curves with the simulation program. The results demonstrated that the interphase should not be neglected and treated as part of the rigid phase, but treated as an independent and separate phase.

As the domain sizes obtained from NMR only and from a combination of SAXS and NMR differed substantially, it was concluded that some of the literature values for the SD coefficients are too large. Consequently, a new average SD coefficient $D_{\mathrm{g}, \text { PS }} \approx 0.38 \pm 0.06 \mathrm{~nm}^{2} \mathrm{~ms}^{-1}$ for glassy PS and a corrected $T_{2}$-based calibration was derived from numerical fitting of the NMR data on the basis of the known domain sizes. The discrepancies and differences, also among the many results from the literature, may be attributable to effects of the magnetic field (low-field vs high-field NMR) and possibly MAS. Work along these lines is currently pursued in our laboratory.

\section{ACKNOWLEDGEMENTS}

We thank Ilja Gunkel and Thomas Thurn-Albrecht (Polymer Physics Group, Univ. Halle) for providing the SAXS data. Funding of this work was provided by the Deutsche Forschungsgemeinschaft (SA982/6-1). We also acknowledge infrastructural support from the European Union (ERDF program).

1 Saalwächter, K. Microstructure and dynamics of elastomers as studied by advanced low-resolution NMR methods. Rubber Chem. Technol. (in press).

2 Schmidt-Rohr, K. \& Spiess, H. W. Multidimensional Solid-State NMR and Polymers (Academic Press, London, 1994).

3 Mellinger, F., Wilhelm, M. \& Spiess, H. W. Calibration of ${ }^{1} \mathrm{H}$ NMR Spin diffusion coefficients for mobile polymers through transverse relaxation measurements. Macromolecules 32, 4686-4691 (1999).

4 Maus, A., Hertlein, C. \& Saalwächter, K. A. Robust proton NMR method to investigate hard/soft ratios, crystallinity, and component mobility in polymers. Macromol. Chem. Phys. 207, 1150-1158 (2006)

5 Mauri, M., Thomann, Y., Schneider, H. \& Saalwächter, K. Spin diffusion NMR at low field for the study of multiphase solids. Solid State Nucl. Magn. Reson. 34, 125-141 (2008).

6 Wang, J. On the determination of domain sizes in polymers by spin diffusion. J. Phys. Chem. 104, 4850-4858 (1996).

7 Buda, A., Demco, D. E., Bertmer, M., Blümich, B., Litvinov, V. M. \& Penning, J. P. General analytical description of spin-diffusion for a three-domain morphology. application to melt-spun nylon 6 fibers. J. Phys. Chem. B 107, 5357-5370 (2003).

8 Voda, M. A., Demco, D. E., Voda, A., Schauber, T., Adler, M., Dabisch, T., Adams, A. Baias, M. \& Blümich, B. Morphology of thermoplastic polyurethanes by ${ }^{1} \mathrm{H}$ spindiffusion NMR. Macromolecules 39, 4802-4810 (2006).

9 Saalwächter, K., Thomann, Y., Hasenhindl, A. \& Schneider, H. Direct observation of interphase composition in block copolymers. Macromolecules 41, 9187-9191 (2008).

10 Thomann, Y., Thomann, R., Hasenhindl, A., Mülhaupt, R., Heck, B., Knoll, K., Steininger, H. \& Saalwächter, K. Gradient interfaces in SBS and SBS/PS blends and their influence on morphology development and material properties. Macromolecules 42, 5684-5699 (2009).

11 McBrierty, V. J. \& Packer, K. J. Nuclear Magnetic Resonance in Solid Polymers (Cambridge University Press, Cambridge, 1993).

12 Rhim, W.- K., Pines, A. \& Waugh, J. S. Time-reversal experiments in dipolar-coupled spin systems. Phys. Rev. B 3, 684-696 (1971).

13 Matsui, S. Solid-state NMR imaging by magic sandwich echoes. Chem. Phys. Lett. 179, 187-190 (1991).

14 Schneider, H. \& Schmiedel, H. Negative time development of a nuclear spin system. Phys. Lett. 30A, 298-299 (1969).

15 Hansen, E. W., Kristiansen, P. E. \& Pedersen, B. Crystallinity of polyethylene derived from solid-state proton NMR free induction decay. J. Phys. Chem. B 102, 5444-5450 (1998).

16 Demco, D. E., Johansson, A. \& Tegenfeldt, J. Proton spin diffusion for spatial heterogeneity and morphology investigations of polymers. Solid State Nucl. Magn. Reson. 4, 13-38 (1995).

$17 \mathrm{Ba}$, Y. \& Ripmeester, J. A. Multiple quantum filtering and spin exchange in solid state nuclear magnetic resonance. J. Chem. Phys. 108, 8589-8594 (1998).

18 Sturniolo, S. \& Saalwächter, K. Breakdown in the efficiency factor of the mixed magic sandwich echo: a novel NMR probe for slow motions. Chem. Phys. Lett. 516, 106-110 (2011).

19 Clauss, J., Schmidt-Rohr, K. \& Spiess, H. W. Determination of domain sizes in heterogeneous polymers by solid-state NMR. Acta Polymer 44, 1-17 (1993) 
20 Saalwächter, K. Proton multiple-quantum NMR for the Study of chain dynamics and structural constraints in polymeric soft materials. Progr. NMR Spectrosc. 51, 1-35 (2007).

21 Jack, K. S., Wang, J., Natansohn, A. \& Register, R. A. Characterization of the microdomain structure in polystyrene-polyisoprene block copolymers by ${ }^{1} \mathrm{H}$ spin diffusion and small-angle X-ray scattering methods. Macromolecules 31, 3282 3291 (1998)

22 Chen, Q. \& Schmidt-Rohr, K. Measurement of the local ${ }^{1} \mathrm{H}$ spin-diffusion coefficient in polymers. Solid State Nucl. Mag. Reson. 29, 142-152 (2006).
23 Vanderhart, D. L. \& Fadden, G. B. Some perspectives on the interpretation of proton NMR spin diffusion data in terms of polymer morphologies. Solid State Nucl. Magn. Reson. 7, 45-66 (1996).

24 Meurer, B. \& Weill, G. Measurement of spin diffusion coefficients in glassy polymers: failure of a simple scaling law. Macromol. Chem. Phys. 209, 212-219 (2008).

25 Roos, M. \& Hempel, G. Monitoring nuclear spin-flip processes and measuring spindiffusion constants via hole burning into the magnetization. Chem. Phys. Lett. 536, 147-154 (2012).

26 Roos, M., Micke, P. \& Hempel, G. Investigation of local static and MAS spin-diffusion coefficients. In preparation. 